\title{
Disruption of sarcolemmal dystrophin and $\beta$-dystroglycan may be a potential mechanism for myocardial dysfunction in severe sepsis
}

\author{
Mara Rúbia N Celes ${ }^{1}$, Diego Torres-Dueñas ${ }^{2,3}$, Lygia M Malvestio ${ }^{1}$, Valdecir Blefari ${ }^{1}$, Erica C Campos ${ }^{1}$, \\ Simone G Ramos ${ }^{1}$, Cibele M Prado ${ }^{1}$, Fernando Q Cunha ${ }^{2}$ and Marcos A Rossi ${ }^{1}$
}

Evidence from our laboratory has shown alterations in myocardial structure in severe sepsis/septic shock. The morphological alterations are heralded by sarcolemmal damage, characterized by increased plasma membrane permeability caused by oxidative damage to lipids and proteins. The critical importance of the dystrophin-glycoprotein complex (DGC) in maintaining sarcolemmal stability led us to hypothesize that loss of dystrophin and associated glycoproteins could be involved in early increased sarcolemmal permeability in experimentally induced septic cardiomyopathy. Male C57BI/6 mice were subjected to sham operation and moderate (MSI) or severe (SSI) septic injury induced by cecal ligation and puncture (CLP). Using western blot and immunofluorescence, a downregulation of dystrophin and $\beta$-dystroglycan expression in both severe and moderate injury could be observed in septic hearts. The immunofluorescent and protein amount expressions of laminin- $\alpha 2$ were similar in SSI and sham-operated hearts. Consonantly, the evaluation of plasma membrane permeability by intracellular albumin staining provided evidence of severe injury of the sarcolemma in SSI hearts, whereas antioxidant treatment significantly attenuated the loss of sarcolemmal dystrophin expression and the increased membrane permeability. This study offers novel and mechanistic data to clarify subcellular events in the pathogenesis of cardiac dysfunction in severe sepsis. The main finding was that severe sepsis leads to a marked reduction in membrane localization of dystrophin and $\beta$-dystroglycan in septic cardiomyocytes, a process that may constitute a structural basis of sepsis-induced cardiac depression. In addition, increased sarcolemmal permeability suggests functional impairment of the DGC complex in cardiac myofibers. In vivo observation that antioxidant treatment significantly abrogated the loss of dystrophin expression and plasma membrane increased permeability supports the hypothesis that oxidative damage may mediate the loss of dystrophin and $\beta$-dystroglycan in septic mice. These abnormal parameters emerge as therapeutic targets and their modulation may provide beneficial effects on future cardiovascular outcomes and mortality in sepsis.

Laboratory Investigation (2010) 90, 531-542; doi:10.1038/labinvest.2010.3; published online 8 February 2010

KEYWORDS: sepsis; septic cardiomyopathy; dystrophin; $\beta$-dystroglycan; dystrophin-glycoprotein complex (DGC); myocardial dysfunction; sarcolemmal permeability

The dystrophin-glycoprotein complex (DGC), which is located in the sarcolemma of cardiac and skeletal muscle cells and concentrated along the plasma membrane in costameric structures that correspond to the $\mathrm{Z}$ bands of the sarcomeres, provides a framework that connects the intracellular cytoskeleton to the extracellular matrix, transmits force between the sarcomere and the plasma membrane to the extracellular matrix and confers structural stability to the cell membrane. ${ }^{1}$ Deficiency of dystrophin results in Duchenne muscular dystrophy (DMD) as well as an associated cardiomyopathy in human patients. The $m d x$ mouse, which lacks dystrophin because of a spontaneous mutation of the murine dystrophin gene, is a model of DMD. This absence or reduction of dystrophin may result in decreased expression of the

\footnotetext{
${ }^{1}$ Department of Pathology (Laboratory of Cellular and Molecular Cardiology), Faculty of Medicine of Ribeirão Preto, University of São Paulo, Ribeirão Preto, São Paulo, Brazil and ${ }^{2}$ Department of Pharmacology (Laboratory of Immunopharmacology), Faculty of Medicine of Ribeirão Preto, University of São Paulo, Ribeirão Preto, São Paulo, Brazil

Correspondence: Professor MA Rossi, MD, PhD, Department of Pathology (Laboratory of Cellular and Molecular Cardiology), Faculty of Medicine of Ribeirão Preto, University of São Paulo, Ribeirão Preto, São Paulo 14049-900, Brazil.

${ }^{3}$ Present address: Universidad Industrial de Santander and Universidad Autónoma de Bucaramanga, Colombia

Received 28 July 2009; revised 28 November 2009; accepted 6 December 2009
} 
remaining DGC components and instability and damage of the sarcolemma, ${ }^{2}$ which leads to muscle fiber degeneration. ${ }^{3}$

Clinical studies have shown that myocardial contractility is reduced in severe sepsis and septic shock in the absence of changes in ventricular preload or afterload. ${ }^{4}$ Cardiac dysfunction has been recognized as a serious manifestations in $\cong 40 \%$ of patients with sepsis, with mortality ranging from 70 to $90 \%$ in contrast with $20 \%$ mortality in septic patients without cardiovascular involvement. ${ }^{5}$

Recent studies from our laboratory give support to the opinion that myocardial structural changes, classifiable as a septic cardiomyopathy, could be responsible for sepsisinduced myocardial dysfunction. ${ }^{5,6}$ The cardiac myofilamentar derangement and dissolution in severe polymicrobial sepsis in mice are heralded by sarcolemmal damage, characterized by increased plasma membrane permeability, which is possibly due to oxidative damage to lipids and proteins. ${ }^{7}$ The critical importance of the DGC in maintaining sarcolemmal stability led us to hypothesize that loss of dystrophin and associated glycoproteins could be involved in early increased sarcolemmal permeability in experimentally induced septic cardiomyopathy. Considering that dystrophin, $\beta$-dystroglycan and laminin- $\alpha 2$ form a mechanical link between the actin cytoskeleton and the extracellular matrix, their expression and localization were evaluated.

\section{MATERIALS AND METHODS Sepsis Model}

Male C57BL/6 mice (20-22 g) were maintained at constant temperature $\left(22 \pm 2{ }^{\circ} \mathrm{C}\right)$ on a $12: 12 \mathrm{~h}$ light-dark cycle. The animal care committee of the Faculty of Medicine of Ribeirão Preto approved the experimental protocol (no. 012/2004), which conforms to the Guide for the Care and Use of Laboratory Animals published by the NIH (Publication no. 85$23,1996)$. Sepsis was induced by cecal ligation and puncture (CLP) with modifications. ${ }^{8}$ In brief, mice were anesthetized with tribromoethanol $(250 \mathrm{mg} / \mathrm{kg}$ body weight i.p.). A midline incision $(1 \mathrm{~cm})$ was made, the cecum ligated with 5-0 cotton thread, and a single puncture made using a 30 -gauge or an 18gauge needle to induce moderate (MSI) and severe (SSI) septic injury, respectively. The cecum was replaced into the abdomen and the incision sutured. Sham-operated mice served as controls. All mice received subcutaneous doses of saline $(50 \mathrm{ml} / \mathrm{kg}$ of body weight) immediately and at $12 \mathrm{~h}$ after procedure to prevent dehydration. The survival rate was monitored every $6 \mathrm{~h}$ for $72 \mathrm{~h}$ after surgery using 10 animals per group (these animals were given subcutaneous doses of saline at 36 and $60 \mathrm{~h}$ after injury to prevent dehydration).

Number of Bacteria in the Peritoneal Cavity and Blood The animals were killed at $6 \mathrm{~h}$ after surgery. For bacterial count in the peritoneal cavity, aliquots of serial log dilutions of the peritoneal aspirate, which were collected under sterile conditions, were plated on Mueller-Hinton agar dishes (Difco Laboratories, Detroit, MI, USA) and incubated at $37^{\circ} \mathrm{C}$ for
$24 \mathrm{~h}$. Colony-forming units (CFUs) were then counted and the results expressed as log of CFU per peritoneal cavity. For bacteremia, the blood was collected under sterile conditions, diluted in sterile saline, and plated on Mueller-Hinton agar dishes (Difco). The number of CFU was counted $24 \mathrm{~h}$ after incubation at $37^{\circ} \mathrm{C}$ and the results expressed as log of CFU per $\mathrm{ml}$ of blood. Five animals per group were evaluated; the results are representative of three different experiments.

\section{Cytokine and Chemokine Measurements}

At $6 \mathrm{~h}$ after CLP, the concentrations of tumor necrosis factor- $\alpha$ (TNF- $\alpha$ ), interleukin $1 \beta$ (IL-1 $\beta$ ), interleukin 6 (IL-6) and monocyte inflammatory protein- $1 \alpha$ (MIP- $1 \alpha$ ) in the peritoneal exudate and serum were determined using a double-ligand enzyme-linked immunosorbent assay (ELISA). In brief, each well of 96-well microtiter plates was coated with antibody anti-TNF $\alpha(2 \mathrm{mg} / \mathrm{ml})$, MIP- $1 \alpha(1 \mathrm{mg} / \mathrm{ml}), \operatorname{IL}-1 \beta(1 \mathrm{mg} / \mathrm{ml})$ or IL-6 $(1 \mathrm{mg} / \mathrm{ml})$, diluted in coating buffer and incubated overnight at $4{ }^{\circ} \mathrm{C}$. Plates were washed, and samples (nondiluted or diluted 1:2) and standards were loaded onto plates. The plates were thoroughly washed, and the appropriate monoclonal anti-cytokine antibody was added. After $1 \mathrm{~h}$, the plates were washed, avidin-peroxidase (diluted 1:5000) was added in each well, and then each plate was thoroughly washed again. The substrate $(0.4 \mathrm{mg}$ of o-phenylenediamine and $0.4 \mathrm{ml}$ of $\mathrm{H}_{2} \mathrm{O}_{2}$ in $1 \mathrm{ml}$ of substrate buffer) was then added and the reaction was stopped with $\mathrm{H}_{2} \mathrm{SO}_{4}(1 \mathrm{M})$, and the optical density (OD) was measured on an ELISA plate scanner (Spectra Max 250; Molecular Devices, Menlo Park, CA, USA) at $490 \mathrm{~nm}$. The results were expressed as picograms of TNF- $\alpha$, IL-1 $\beta$, IL-6 and MIP-1 $\alpha$ per milliliter of supernatant or serum. Recombinant murine TNF- $\alpha$, IL- $1 \beta$, IL- 6 and MIP- $1 \alpha$ standard curve were used to calculate the cytokine concentrations. Five animals per group were evaluated; the results are representative of three different experiments.

\section{Measurement of Mean Carotid Blood Pressure}

Mean carotid artery blood pressure was obtained in anesthetized animals (tribromoethanol $250 \mathrm{mg} / \mathrm{kg}$ i.p.) at 6 , 12 and $24 \mathrm{~h}$ after surgery $(n=6$ animals per group each time). A polyethylene catheter (PE-10) filled with heparinized saline was introduced and positioned in the right carotid artery and exteriorized in the neck. Immediately after surgical preparation, when hemodynamic was stable, mean carotid pressure (MCP) was recorded connecting the catheter to a pressure transducer PowerLab (AD Instruments, Castle Hill, Australia). The carotid blood pressure of anesthetized animals weighing $22 \mathrm{~g}$ simulating the time 0 of the experiment immediately after CLP or sham surgery was also measured. The results were expressed as $\mathrm{mm} \mathrm{Hg}$.

\section{Immunofluorescence}

For immunofluorescence study, heart fragments from SSI, MSI and sham-operated mice ( $n=5$ animals/group) were excised $24 \mathrm{~h}$ after surgery, immediately sectioned into 
anterior and posterior halves, frozen in isopentane chilled in liquid nitrogen, and stored accordingly at $-80^{\circ} \mathrm{C}$. Immunolabeling was performed ( $5 \mu$ m-thick sections) using primary antibodies to dystrophin (rabbit polyclonal antibody anti-dystrophin, 1:200; Santa Cruz Biotechnology, Santa Cruz, CA, USA), $\beta$-dystroglycan (goat polyclonal antibody anti- $\beta$-dystroglycan, 1:400; Vector Laboratories, Burlingame, CA, USA) or merosin (laminin- $\alpha 2$ ) (goat polyclonal antibody anti-laminin- $\alpha 2$, 1:100; Santa Cruz Biotechnology). Fluoresceinconjugated secondary antibodies anti-rabbit IgG, diluted 1:400 or anti-goat IgG, diluted 1:400 (Vector Laboratories) were used. Omission of the primary antibodies served as negative controls. For quantification of the mean area (\%) and fluorescence intensity (OD) of immunoreactivity particles of dystrophin, $\beta$ dystroglycan and laminin- $\alpha 2,5-10$ randomly selected images of transversely oriented myofibers of $0.0093 \mathrm{~mm}^{2}$ were captured from each of five hearts per group and analyzed using the Leica Qwin software (Leica Imaging Systems, Cambridge, UK) in conjunction with Leica DMR microscope (Leica Systems, Wetzlar GmbH, Germany).

Some sections were also labeled with phalloidin complexed to rhodamine (Alexa Fluor 594 phalloidin, Molecular Probes, Eugene, OR, USA) for visualization of actin. Immunofluorescent staining for albumin was performed to evaluate the structural integrity of the sarcolemma of cardiomyocytes using fluoresceinated antibody to albumin (goat polyclonal antibody anti-mouse albumin FITC conjugated; Bethyl Laboratories, Montgomery, TX, USA; 1:400). Sections were stained with wheat germ agglutinin (WGA) conjugated with Texas red (Vector Laboratories) as a selective probe for plasma membrane integrity. WGA is a carbohydrate-binding protein that selectively recognizes sialic acid and $\mathrm{N}$-acetylglucosaminyl sugar residues, which are predominantly found on the plasma membrane. To determine whether the administration of peroxide dismutase (SOD), which is a highly selective superoxide scavenger, could influence the expression of dystrophin and/or sarcolemmal permeability in polymicrobial sepsis, an additional experiment was performed: sham and SSI mice were treated with SOD (2000 units $/ \mathrm{kg}$ of body weight, $100 \mu \mathrm{l} / \mathrm{animal}$ i.p., immediately after surgery; Sigma); sham and SSI mice were also treated with saline as placebo $(100 \mu \mathrm{l} /$ animal i.p., immediately after surgery). To prevent dehydration, all mice received saline subcutaneously $(50 \mathrm{ml} / \mathrm{kg}$ of body weight) immediately and $12 \mathrm{~h}$ after septic injury. Sections were examined using a Leica DM6000 B microscope equipped with Leica AF6000 Deconvolution (Leica Microsystems).

\section{Western Blot Analysis}

To determine the amount of dystrophin, $\beta$-dystroglycan and laminin- $\alpha 2$ in septic (MSI and SSI) and sham-operated hearts, homogenates of left and right ventricles ( $n=3-5$ animals/group) were analyzed using immunoblotting at $24 \mathrm{~h}$ after surgery. Fresh hearts were washed in cold PBS, and the left and right ventricles were isolated and homogenized in extraction buffer and protease inhibitor cocktail (Sigma-Aldrich, Saint Louis, MO, USA). Total heart protein ( $40 \mu \mathrm{g}$ protein/well) was resolved on a 5 or $10 \%$ SDS-PAGE, and transferred to nitrocellulose membrane (Hybond-ECL; Amersham Pharmacia Biotech, Amersham, UK). The membranes were blocked with $5 \%$ skimmed milk/TBS-T for $24 \mathrm{~h}$, and incubated overnight at $4{ }^{\circ} \mathrm{C}$ with the primary antibodies (rabbit polyclonal anti-dystrophin, 1:750, goat polyclonal anti- $\beta$-dystroglycan, 1:500; Santa Cruz Biotechnology and goat polyclonal anti- $\alpha$-tubulin, 1:1000 Santa Cruz Biotechnology). The blots were washed and incubated with HRP-conjugated secondary antibodies: rabbit anti-goat IgG, diluted 1:20 000 and goat anti-rabbit IgG, diluted 1:10 000 (Vector Laboratories), for $1 \mathrm{~h}$ at room temperature. The membranes were washed, developed using ECL (Amersham Pharmacia Biotech) and exposed to hyperfilm ECL (Amersham Pharmacia Biotech). Analysis of ECL images was performed using the public domain Image J program (developed at the National Institutes of Health and available at http://rbs.info.nih.gov/nih-image/), using the 'Gel Analysis' functions. Result of the analysis is a value for each band, which is proportional to the integrated density value (IDV) of that band, which corresponds to arbitrary units (AUs). $\propto$-Tubulin was used to determine approximately equivalent loading conditions.

\section{Statistical Analysis}

Multiple comparisons were made using one-way ANOVA followed by Tukey or Bonferroni post-tests. Comparisons of two groups were made using Student's $t$-test. A $P<0.05$ was considered statistically significant. The survival rate was expressed as the percentage of live animals. The survival rates were constructed using the Kaplan-Meier method, and differences in mortality were compared using the log-rank-test. A $P<0.05$ was considered statistically significant.

\section{RESULTS}

\section{The Experimental Model}

\section{Survival rate in sepsis}

Figure 1a shows the survival rate of the mice submitted to sham operation, MSI and SSI until $72 \mathrm{~h}$ after surgery. The septic mice showed reduced activity, piloerection, and exudation around the eyes and nose at $24 \mathrm{~h}$ after CLP surgery. In contrast, the sham animals showed full recovery from anesthesia and surgery, and had no detectable signs of disease. The SSI mice showed $20 \%$ mortality at $12 \mathrm{~h}$ after injury, increasing to $50 \%$ at $24 \mathrm{~h}$ after cecal puncture, reaching $90 \%$ at $48 \mathrm{~h}$ after CLP and remaining steady until $72 \mathrm{~h}$ after surgery. The MSI mice showed $10 \%$ mortality at $24 \mathrm{~h}$ after injury, increasing to $30 \%$ mortality at $72 \mathrm{~h}$. Shamoperated animals presented a $100 \%$ survival rate through the analyzed period of $72 \mathrm{~h}$.

\section{Mean carotid blood pressure}

Figure $1 \mathrm{~b}$ shows the mean values of carotid blood pressure at $0,6,12$ and $24 \mathrm{~h}$ after CLP and sham operation. Carotid blood pressure simulating the time 0 of the experiment, immediately after surgery, was similar for sham, MSI and SSI 

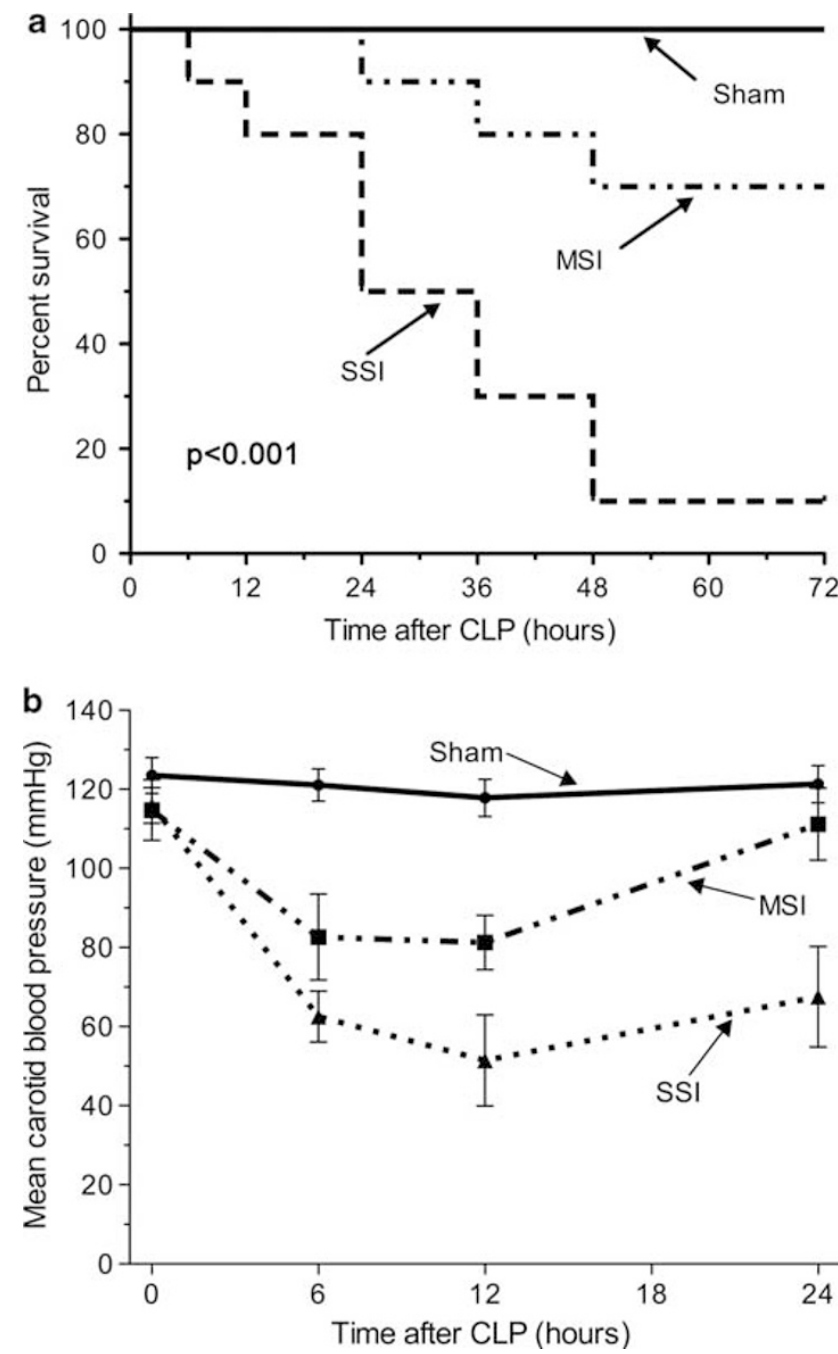

Figure 1 Mortality rate and carotid blood pressure. Curves of actuarial mortality of mice submitted to sham operation, moderate (MSI) and severe (SSI) septic injury (a). The survival rate was determined daily until $72 \mathrm{~h}$ after surgery ( $n=10$ animals per group; the results are representative of three different experiments). Mean carotid blood pressure $(\mathrm{mm} \mathrm{Hg})$ at $0,6,12$ and $24 \mathrm{~h}$ after CLP or sham operation (b). The number of animals is six for each group at each time. Data are expressed as mean \pm s.d. $P<0.001$ indicates the level of difference in comparison with sham control.

mice $(123.50 \pm 4.53,114.70 \pm 7.67$ and $115.90 \pm 4.47 \mathrm{~mm} \mathrm{Hg}$, respectively). The values of blood pressure were significantly lower in SSI and MSI mice undergoing CLP at $6 \mathrm{~h}$ (48.3 and $31.7 \%$ reduction, respectively) and $12 \mathrm{~h}$ (56.3 and $31 \%$ reduction, respectively) after injury when compared with sham-operated controls. At $24 \mathrm{~h}$ after injury, the carotid blood pressure of SSI mice remained lower compared with that observed in MSI and control mice (39.3 and 44.3\% reduction, respectively) as long as the mean arterial pressures of MSI and sham-operated mice were not statistically different.

\section{Local and systemic infection}

At $6 \mathrm{~h}$ after CLP surgery, the bacterial count in the peritoneal cavity and blood (Figures 2a and b) was performed. The mean number of bacteria in the peritoneal cavity of SSI mice was higher than that of MSI and sham control animals. MSI mice showed a small number of bacteria in the peritoneal cavity, statistically different from sham-operated mice. Bacterial load in blood samples obtained from SSI mice presented higher number in comparison with MSI and sham groups. MSI mice showed a mild bacteremia statistically different from the negative bacteremia of sham controls.

\section{Local and systemic inflammatory response}

Figure 2 shows the levels of TNF- $\alpha$, IL- $1 \beta$, IL- 6 , and MIP- $1 \alpha$ in peritoneal exudate and serum from mice submitted to CLPinduced sepsis or sham procedure at $6 \mathrm{~h}$ after surgery. SSI mice presented markedly increased concentrations of TNF $\alpha$, IL- $1 \beta$, IL- 6 and MIP- $1 \alpha$ in the peritoneal cavity and serum when compared with MSI and sham-operated mice. MSI mice presented IL- $1 \beta$ and IL-6 peritoneal exudate levels similar to those of SSI animals, much higher than the concentrations in shamoperated mice. The TNF- $\alpha$ levels in peritoneal exudate and serum of MSI mice were not different from those in sham controls. MSI mice showed serum IL- $1 \beta$ levels similar to those of controls, whereas the levels of IL- 6 were significantly higher in comparison with sham-operated animals.

The peritoneal and serum MIP- $1 \alpha$ concentrations were markedly increased in SSI group when compared with MSI and sham-operated mice. The concentrations of MIP- $1 \alpha$ in the MSI mice were significantly higher in the peritoneal exudate when compared with sham animals, whereas the serum levels of this chemokine were not different.

\section{Western blot analysis of dystrophin, $\beta$-dystroglycan and laminin- $\alpha 2$}

Figures $3 \mathrm{a}$ and $4 \mathrm{a}$ show the amounts of dystrophin and $\beta$-dystroglycan at $24 \mathrm{~h}$ after sepsis (MSI and SSI) or sham operation expressed in AUs and representative blots. The amount of dystrophin (Figure 3a) in SSI mice heart ventricles (2812.0 \pm 351.9 AUs) was markedly reduced when compared with MSI (4686.0 $\pm 474.3 \mathrm{AUs})$ and sham-operated $(5642 \pm 331.4 \mathrm{AUs})$ heart ventricles. These values represent a reduction of 40 and $50 \%$ in MSI and sham-operated mice, respectively. The amount of $\beta$-dystroglycan (Figure 4a) was markedly decreased in SSI (2272.0 \pm 240.6 AUs) when compared with MSI $(3898.0 \pm 449.7$ AUs) and sham-operated ( $3858.0 \pm 430.6 \mathrm{AUs})$ heart ventricles. These values represent a reduction of 41 and $42 \%$ in MSI and sham-operated mice, respectively. The amounts of laminin- $\alpha 2$ at $24 \mathrm{~h}$ after surgery were similar in the hearts of animals in the three groups studied (data not shown).

\section{Immunofluorescent analysis of dystrophin, $\beta$-dystroglycan and laminin- $\alpha 2$}

The immunofluorescent signals for dystrophin and $\beta$-dystroglycan were strikingly reduced in SSI hearts (Figures 3c and $4 \mathrm{c}$, respectively), whereas sham-operated hearts showed abundant signal for these proteins (Figures $3 \mathrm{~b}$ and $4 \mathrm{~b}$, respectively), similar to the expression in MSI hearts (data not 

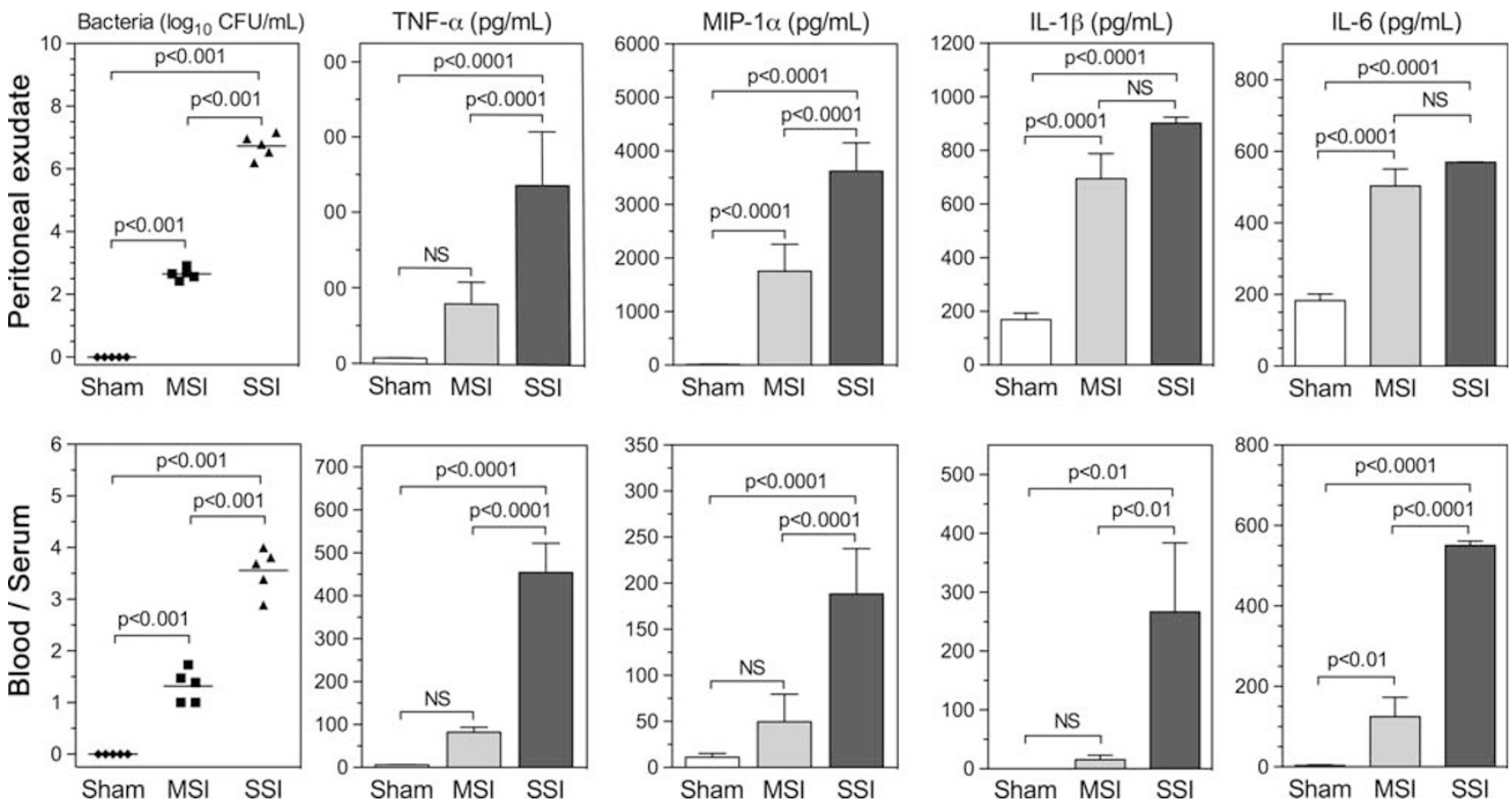

Figure 2 Bacterial count and inflammatory response. Peritoneal cavity infection and bacteremia (a, b). The number of CFUs in peritoneal cavity (a) and serum (b) of mice submitted to sham operation, MSI and SSI mice were evaluated at $6 \mathrm{~h}$ after surgery. Results are expressed as mean number of CFU/cavity or CFU/ml of blood ( $n=5$ animals per group; the results are representative of three different experiments). The mean number of CFU in the peritoneal cavity of SSI mice was higher than that of MSI and sham control animals. The levels of TNF- $\alpha$, IL-1 IL-1 $\beta$, IL- 6 and MIP- $1 \alpha$ were quantified in peritoneal exudate and serum at $6 \mathrm{~h}$ after surgery in sham-operated, MSI and SSI mice ( $n=5$ animals per group; the results are representative of three different experiments). Data are expressed as mean \pm s.d. NS, not significant.

shown). In contrast, the analysis of immunofluorescence for laminin- $\alpha 2$ did not show statistical difference in the fluorescent signal in SSI hearts (Figure 5b) when compared with those of sham-operated and MSI hearts (Figure 5a).

The mean area (\%) and fluorescent intensity (OD) of dystrophin in the left ventricles of SSI mice $(10.5 \pm 1.59 \%$ and $19.9 \pm 0.45 \mathrm{OD}$, respectively) were strikingly decreased, representing a reduction of 40 and $41 \%$, respectively, when compared with the values in the sham-operated hearts $(17.5 \pm 3.09 \%$ and $33.8 \pm 1.40 \mathrm{OD}$, respectively; Figures $3 \mathrm{~d}$ and e). The mean area $(\%)$ and fluorescent intensity $(\mathrm{OD})$ of $\beta$-dystroglycan in the left ventricles of SSI mice $(10.3 \pm 0.31 \%$ and $18.5 \pm 0.61 \mathrm{OD}$, respectively) were decreased, representing a reduction of 21 and $23 \%$, respectively, in comparison with the values in the shamoperated hearts $(13.0 \pm 0.76 \%$ and $24.0 \pm 1.20 \mathrm{OD}$, respectively; Figures $4 \mathrm{~d}$ and e). The mean area (\%) and fluorescent intensity (OD) of laminin- $\alpha 2$ in the hearts of SSI mice $(42.52 \pm 7.86 \%$ and $92.06 \pm 16.96 \mathrm{OD}$, respectively), did not show significant reduction when compared with the values observed in the hearts of sham-operated mice $(46.20 \pm 5.45 \%$ and $113.5 \pm 4.73 \mathrm{OD}$, respectively; Figure 5).

\section{Sarcolemmal Permeability Evaluated by Albumin Staining}

In controls, albumin was mainly localized in the interstitial space as a delicate network and in the vascular lumina. In SSI hearts, albumin labeling was more intense in the interstitial space compared with sham controls and, importantly, spread large blocks of myocytes became positive showing intracytoplasmic albumin labeling. Albumin-WGA double staining clearly depicted the cardiac myofibers in the two groups and spread blocks of muscle cells showing a uniformly diffuse pattern of green fluorescent cytoplasmic albumin staining and the more intense green fluorescence of albumin in the interstitial space in SSI hearts (Figure 6). In MSI myocardium, albumin was mainly localized in the interstitial space, and was much less intense compared with SSI myocardium, with a few spread blocks of myocytes showing faint albumin staining in the cytoplasm (data not shown).

The distribution of dystrophin in myocytes showing increased sarcolemmal permeability was assessed in sections labeled using both albumin (green fluorescence) and dystrophin (red fluorescence) in SSI and control hearts (Figure 7). Albumin-dystrophin double labeling in septic hearts clearly showed that SSI mice presented accumulation of albumin mainly in spread blocks of cardiomyocytes depleted of sarcolemmal dystrophin and in the interstitium. In SSI mice treated with SOD, the expression of intracytoplasmic and interstitial green fluorescent albumin was significantly reduced whereas the reduced expression of dystrophin was significantly attenuated. 


\section{DISCUSSION}

The CLP model of sepsis/septic shock in rodents, which is considered the gold standard for sepsis research, has been widely used, producing a clinical situation similar to that observed in human sepsis. ${ }^{9}$ Many of the attributes of an appropriate model were satisfied: it is polymicrobial, has
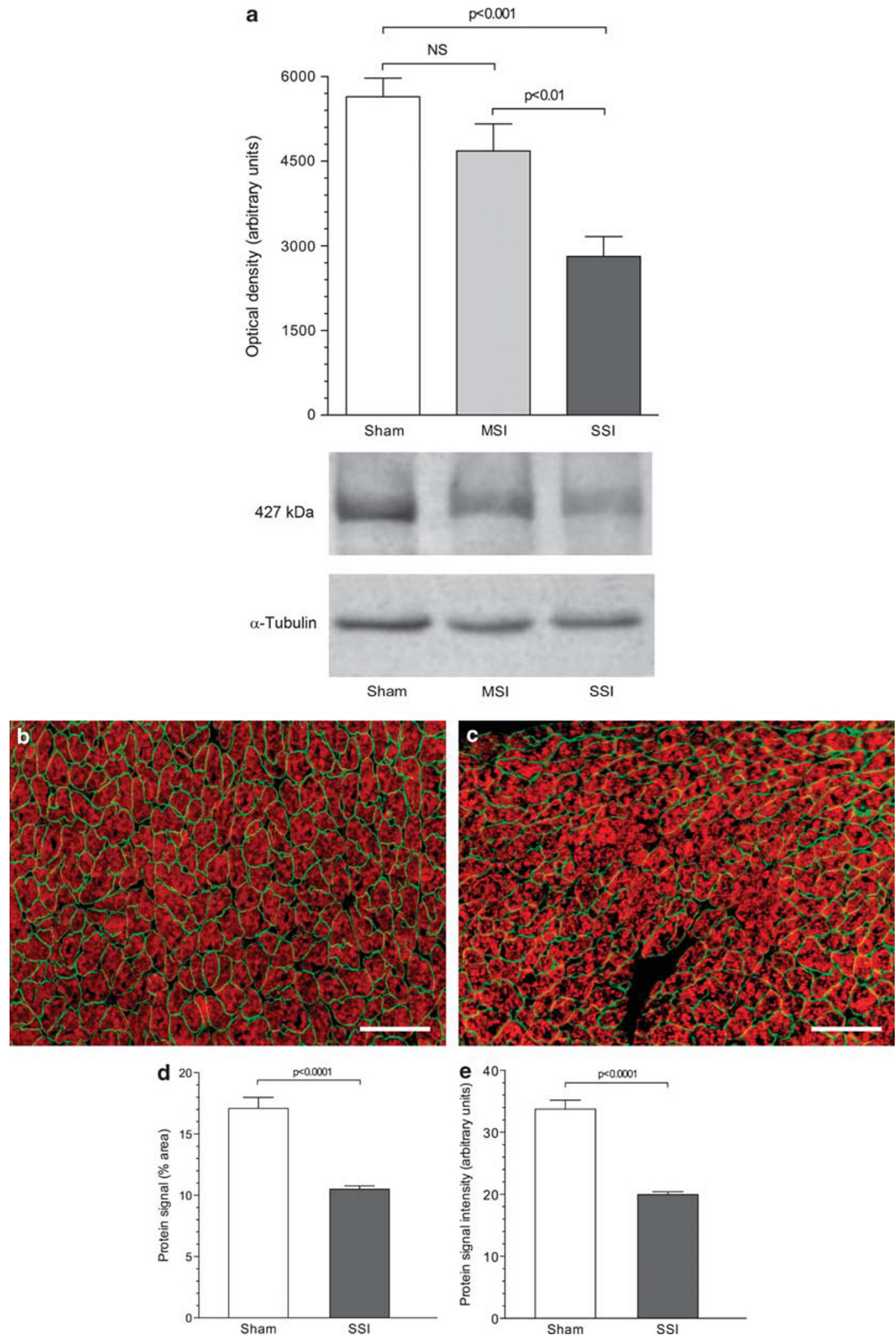
focal infection origin, produced septicemia, induced high levels of cytokines and chemokine in peritoneal exudate and serum and circulatory shock.

The peritoneal exudate and serum level TNF- $\alpha$, IL- $1 \beta$, IL-6 and MIP- $1 \alpha$ levels measured $6 \mathrm{~h}$ after surgery were significantly higher in SSI mice when compared when sham controls. At the same time, only the peritoneal exudate IL- $1 \beta$, IL- 6 and MIP- $1 \alpha$ levels were higher in MSI mice in comparison with sham-operated controls, whereas the TNF- $\alpha$ concentrations were similar; only the serum IL-6 levels in MSI mice were increased when compared with control, whereas the serum TNF $\alpha$, IL- $1 \beta$ and MIP- $1 \alpha$ concentrations were not different. In the past few decades, it has been observed that several mediators of inflammation tend to become elevated during sepsis. Circulating cytokines, such as TNF- $\alpha$, IL- $1 \beta$ and IL- 6 , have been suggested as causative factors of myocardial depression in sepsis. ${ }^{10,11}$ Consonantly, MIP- $1 \alpha$, a member of the CC chemokines family, has been shown to exert a significant activating and a chemotactic effect on macrophages, including their release of TNF- $\alpha$, and, unlike other members of the family, seems to regulate polymorphonuclear function. ${ }^{12,13}$

At $24 \mathrm{~h}$ after injury, the mean carotid blood pressure of SSI mice remained very low as long as the mean carotid blood pressure of MSI returned to a normal value comparable to that of sham animals. The parameters analyzed at $6 \mathrm{~h}$ after CLP represent the first effects of the injurious influence at the molecular or biochemical levels. The parameters analyzed at $24 \mathrm{~h}$ after CLP, and the amounts of dystrophin, $\beta$-dystroglycan and laminin- $\alpha 2$ evaluated using western blot and immunofluorescence, represent the early morphological consequences of the injury. All stresses and injurious influences exert their effects first at the molecular or biochemical level. There is a lag between the stress and the morphological changes of cell injury or death. The duration of this delay vary according to the sensitivity of the methods used to detect the changes. The morphological changes in this study were only detected in the late periods of the sepsis model used in this study, that is, at $24 \mathrm{~h}$ after injury.

Western blot analysis of dystrophin expression presented a marked decrease of this structural protein in the hearts of mice submitted to SSI when compared with mice submitted to MSI and sham operation (reduced by 40 and $50 \%$, respectively). Dystrophin protein expressions in MSI and sham hearts were similar. In addition, western blot analysis of $\beta$-dystroglycan showed that the expression of this transmem- brane protein was markedly decreased in the hearts of SSI in comparison to its expression in the hearts of MSI and shamoperated mice (reduced by 41 and $42 \%$, respectively). MSI and sham-operated hearts showed similar $\beta$-dystroglycan protein expression. The expressions of laminin- $\alpha 2$ were similar in the three groups studied.

The immunofluorescence study allowed us to characterize morphologically the loss of both dystrophin and $\beta$-dystroglycan in SSI induced by CLP in mice. More specifically, immunofluorescence revealed that the areas and the mean intensities (OD) of both dystrophin and $\beta$-dystroglycan expressions decreased compared with the expressions of both proteins in sham hearts. The mean area of dystrophin decreased by $40 \%$ whereas the mean OD decreased by $41 \%$ when compared with sham hearts. The mean area and mean OD of $\beta$-dystroglycan decreased less than those of dystrophin, 21 and $23 \%$, respectively, compared with sham hearts. In contrast, the immunofluorescent analysis for laminin- $\alpha 2$ did not showed statistical difference in SSI hearts when compared with sham-operated hearts.

The functional consequences of our findings are not clear at this moment, because we do not know whether the loss of both dystrophin and $\beta$-dystroglycan necessarily establish a direct cause/effect relationship with myocardial contractile dysfunction in the hearts of mice with severe sepsis/septic shock. However, the occurrence of hypotension and circulatory shock in mice with severe sepsis suggests myocardial depression.

Reduction or absence of dystrophin and $\beta$-dystroglycan implies rupture of the mechanical linkage that attaches the actin-based subsarcolemmal cytoskeleton and the sarcomeres to the sarcolemma, which may be related to impairment of the contractile force transmission. Numerous studies have focused on dystrophin with its associated membrane glycoprotein complex and their physiopathologic role on myocardial contractile performance. The absence of dystrophin constitutes the structural basis in the hearts of patients with DMD as well as in $m d x$ mice associated with the development of dilated cardiomyopathy. ${ }^{14}$ The lack of dystrophin in $m d x$ mice severely compromises the mechanical integrity of cardiomyocytes, which are more vulnerable to workloadinduced damage. ${ }^{15}$ In addition, loss of dystrophin has been linked with end-stage cardiomyopathies and has been proposed as a common pathway for contractile dysfunction in the failing myocardium and progression of advanced heart failure. ${ }^{16}$ Recent study shows that antisense

Figure 3 Analysis of dystrophin. Western blot analysis of dystrophin (a). The amount of dystrophin in the MSI, SSI and sham operation group were measured at $24 \mathrm{~h}$ after surgery and expressed in arbitrary units (AUs). $\alpha$-Tubulin was used to determine equivalent loading conditions. Results ( $n=3-5$ per group) are representative of three different experiments. Immunofluorescence analysis of dystrophin (b-e). The immunofluorescent signal for dystrophin is strikingly reduced in SSI myocardium (c, right panel) in comparison with the immunofluorescent signal in sham-operated myocardium (b, left panel). The mean area (\%) (d) and fluorescent intensity (OD) (e) of dystrophin in the heart left ventricles of SSI mice are markedly decreased when compared with the values in the left ventricles of sham-operated heart mice. The graphs show the batches of data of percent area (right, bottom panel) and optical density (left, bottom panel) of dystrophin signals ( $n=5$ ventricles hearts per group). Scale bars indicate $50 \mu \mathrm{m}$. NS, not significant. 
therapeutic dystrophin restoration to almost normal levels in cardiac skeletal muscle in $m d x$ mice prevents the development of cardiac pump failure and leads to increase in muscle strength. ${ }^{17}$ To date, no form of muscular dystrophy has been linked with the dystroglycan gene itself in human patients. Perhaps this can be explained by the demonstration that $p<0.001$

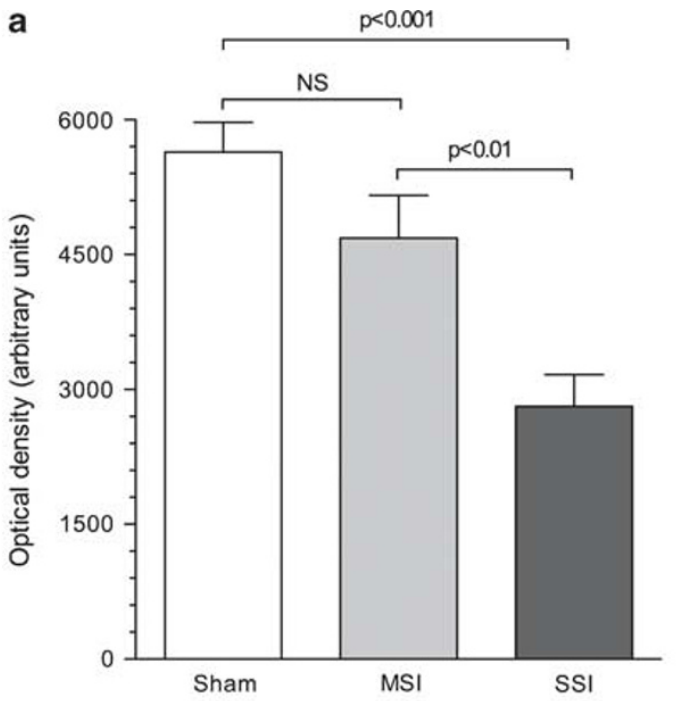

$43 \mathrm{kDa}$
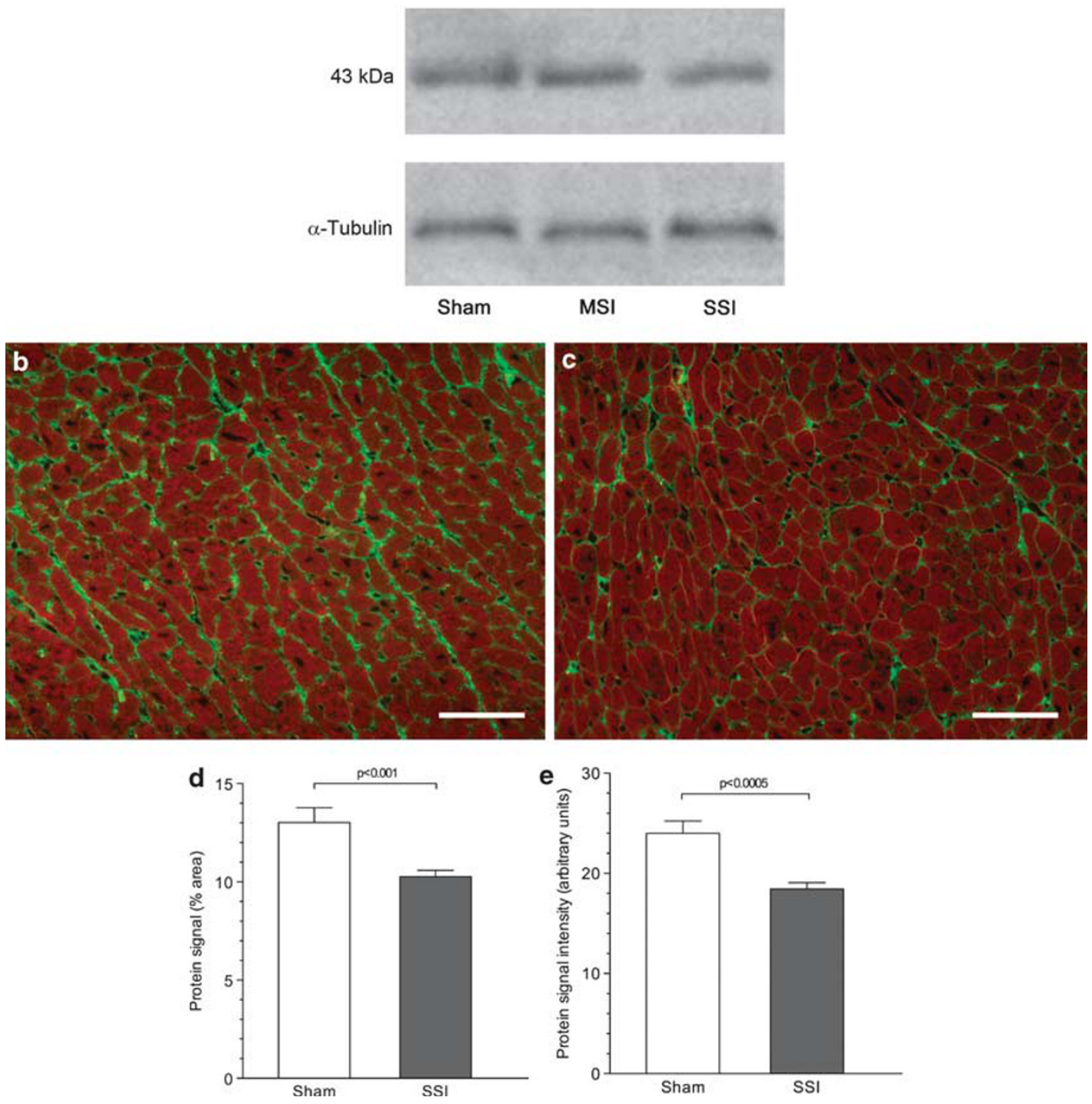
dystroglycan is essential for early embryonic development in mice, with its absence resulting in death. ${ }^{18}$ In DMD patients and $m d x$ mice, there is a drastic reduction of dystroglycan protein in the sarcolemma affecting the connection between basement membranes and the cytoskeleton and the interaction with laminin- $\alpha 2$ in the muscle cell basement membrane. ${ }^{19,20}$ Moreover, it has been shown that the proteolytic processing of $\beta$-dystroglycan is involved in the muscle degeneration process of cardiomyopathic hamsters. $^{21}$

The detection of intracellular albumin to evaluate plasma membrane permeability provided evidence of sarcolemmal damage in SSI hearts, confirming previous observations from our laboratory. ${ }^{7}$ This study showed that sarcolemmal permeability increase is an early event in myocardial injury in severe sepsis possibly due to oxidative damage to lipids and proteins. Importantly, in the present investigation the SSI hearts presented accumulation of albumin in spread blocks of cardiomyocytes depleted of dystrophin. Increased sarcolemmal permeability indicates functional impairment of the DGC in severe sepsis. Moreover, an increased accumulation of interstitial albumin, reflecting increased microvascular permeability, could be depicted. It is notable, however, that the disruption of dystrophin- $\beta$-dystroglycan in the
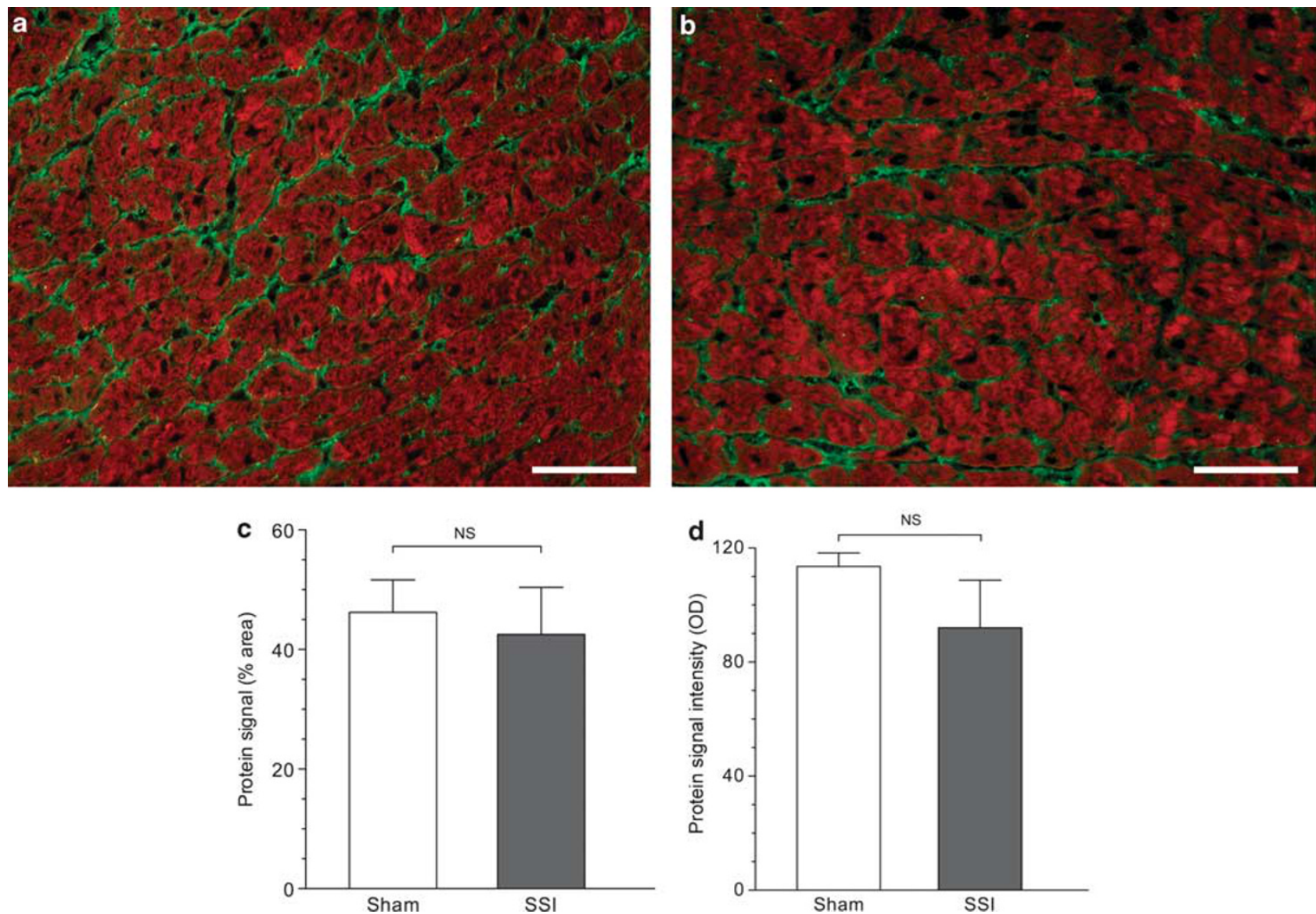

Figure 5 Immunofluorescence analysis of laminin- $\alpha 2$. The immunofluorescent signal for laminin- $\alpha 2$ was similar in SSI myocardium (b, right panel) in comparison with the immunofluorescent signal in sham-operated myocardium (a, left panel). The mean area (\%) (c) and fluorescent intensity (optical density (OD) expressed in arbitrary units) (d) of laminin- $\alpha 2$ in the heart left ventricles of SSI mice were not different from the values in the left ventricles of sham-operated hearts. The graphs show the batches of data of percent area and optical density of laminin- $\alpha 2$ signals ( $n=5$ ventricles hearts per group). Scale bars indicate $50 \mu \mathrm{m}$. NS, not significant.

Figure 4 Analysis of $\beta$-dystroglycan. Western blot analysis of $\beta$-dystroglycan (a). The amount of $\beta$-dystroglycan in the MSI, SSI and sham operation group were measured at $24 \mathrm{~h}$ after surgery and expressed in arbitrary units (AUs). $\alpha$-Tubulin was used to determine equivalent loading conditions. Results ( $n=3-5$ per group) are representative of three different experiments. Immunofluorescence analysis of $\beta$-dystroglycan (b-e). The immunofluorescent signal for $\beta$-dystroglycan is reduced in SSI myocardium (c, right panel) in comparison with the immunofluorescent signal in sham-operated myocardium (b, left panel). The mean area (\%) (d) and fluorescent intensity (optical density (OD) expressed in arbitrary units) (e) of $\beta$-dystroglycan in the heart left ventricles of SSI mice are decreased when compared with the values in the left ventricles of sham-operated heart mice. The graphs show the batches of data of percent area and OD of $\beta$-dystroglycan signals ( $n=5$ ventricles hearts per group). Scale bars indicate $50 \mu \mathrm{m}$. NS, not significant. 

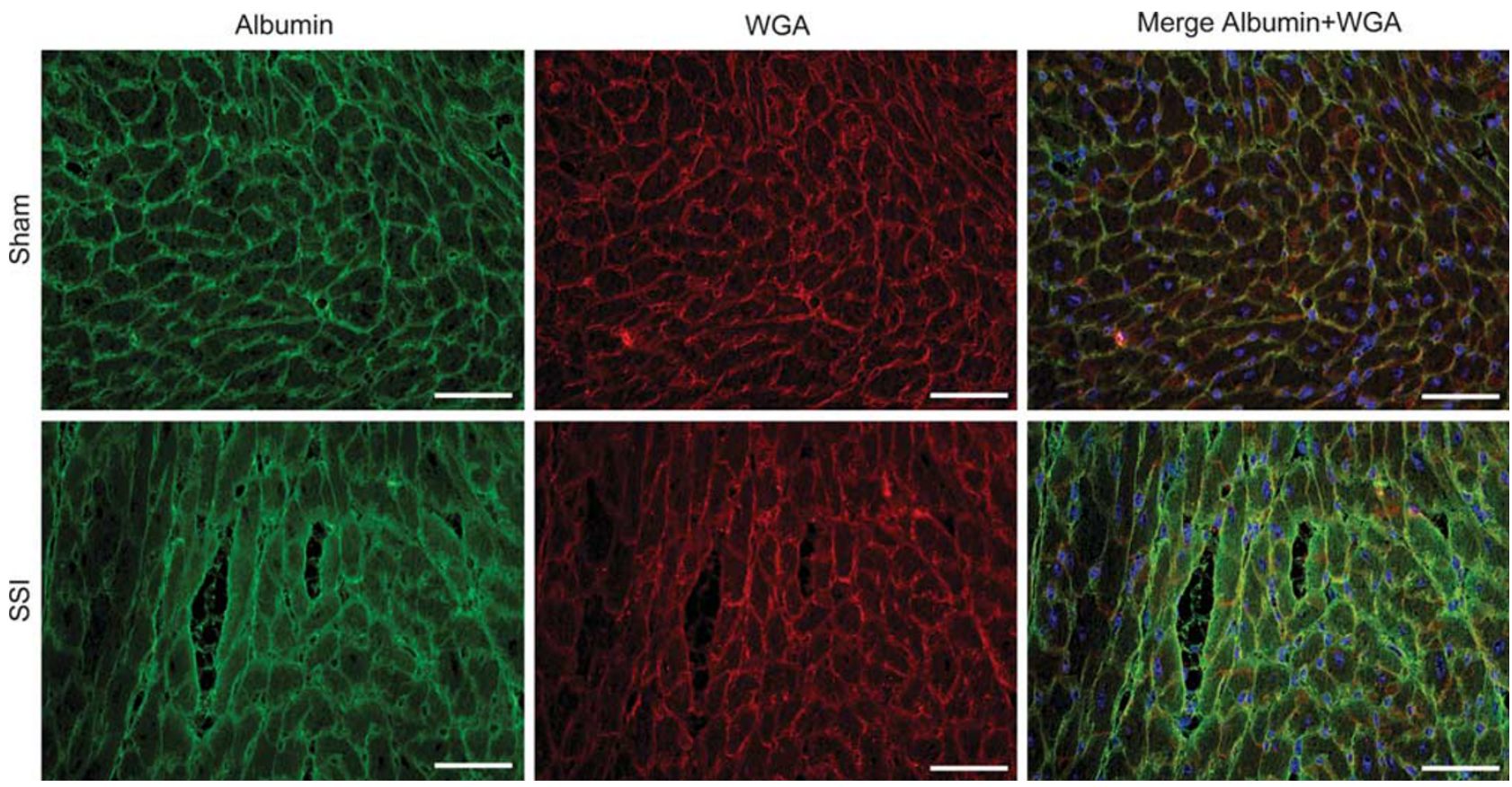

Figure 6 Sarcolemmal permeability evaluated using albumin-WGA double staining. Immunolabeling for albumin (green fluorescence), plasma membrane labeling with WGA (red fluorescence) and nuclear staining with DAPI (blue fluorescence) was evaluated at $24 \mathrm{~h}$ after surgery. Albumin-WGA double staining clearly depicted the cardiac myofibers in the two groups and spread blocks of muscle cells showing a uniformly diffuse pattern of green fluorescent cytoplasmic albumin staining and the more intense green fluorescence of albumin in the interstitial space in SSI hearts. Scale bars $=50 \mu \mathrm{m}$.

sarcolemma occurs without disintegration of the plasma membrane, as evidenced by preserved WGA staining in SSI hearts, possibly indicating reversible damage, at least at this point of the study. The accumulation of interstitial fluorescent albumin was markedly reduced. Moreover, the marked reduction of sarcolemmal permeability with the administration of a superoxide scavenger indicates the involvement of free radicals in its genesis. There is good evidence that endotoxemia, sepsis and septic shock are associated with the generation of reactive oxygen species (ROS), including superoxide anion $\left(\mathrm{O}_{2}^{-}\right)$and hydrogen peroxide $\left(\mathrm{H}_{2} \mathrm{O}_{2}\right),{ }^{22}$ which can cause endothelial damage and increased microvascular permeability, recruitment of neutrophils at sites of inflammation, lipid peroxidation, DNA damage and formation of peroxynitrite $\left(\mathrm{ONOO}^{-}\right) .^{23}$ Of the DGC proteins, dystrophin forms a strong mechanical link between the sarcolemma and the costameric cytoskeleton in the cardiac muscle cells stabilizing the sarcolemma from stresses developed during muscle contraction. $^{24,25}$ Cardiomyocytes lacking dystrophin are abnormally vulnerable to mechanical stress-induced injury, with loss of sarcolemmal integrity and increased fragility and permeability. ${ }^{26,27}$ In analogy to DMD ${ }^{28}$ the loss of dystrophin led to a significant reduction of its binding transmembranous partner $\beta$ dystroglycan, which is essential for the structure and stability of the costamere, which is the skeleton of the muscle membrane. ${ }^{29}$ Moreover, high levels of proinflammatory cytokines may exacerbate the membrane damage caused by dystrophin deficiency in mice submitted to SSI. ${ }^{30}$
An important question is to elucidate 'what is the mechanism of the reduction of dystrophin and $\beta$-dystroglycan expression in the hearts of mice submitted to severe CLP sepsis?' First, considering that one of the key features in sepsis is a heterogeneous distribution of blood flow, ${ }^{31-33}$ with certain microvascular beds underperfused whereas others normal or hyperperfused, ${ }^{25}$ the dystrophin and $\beta$-dystroglycan expression may be attributed to global ischemia. Among the distinctive group of proteins that link the intracellular and extracellular milieus and confer structural stability to the cell membrane, dystrophin is reported to be the most sensitive to ischemic insult and a useful ischemic index. . $^{2,34}$ Acute ischemia is known to cause loss of immunostained dystrophin and corresponding depression of dystrophin in cardiomyocytes in vitro. ${ }^{35}$ Similarly, isolated rabbit cardiomyocytes subjected to late in vitro ischemia shows loss of cytoskeletal $\beta$-dystroglycan with corresponding appearance of its degradation product. ${ }^{36}$ Second, endogenous or exogenous proteases could cause dystrophin expression loss. It has been shown that enteroviral protease $2 \mathrm{~A}$ (Coxsackievirus B3) directly cleaves dystrophin in experimentally induced infection in cardiomyocytes in culture and in infected hearts, leading to impaired dystrophin function. ${ }^{37,38}$ The plasma membrane damage can lead to increased intracellular calcium; the consequences of cytosolic calcium may include activation of proteases, such as calpain, which can degrade cytoskeletal and membrane proteins, including dystrophin. ${ }^{39}$ 

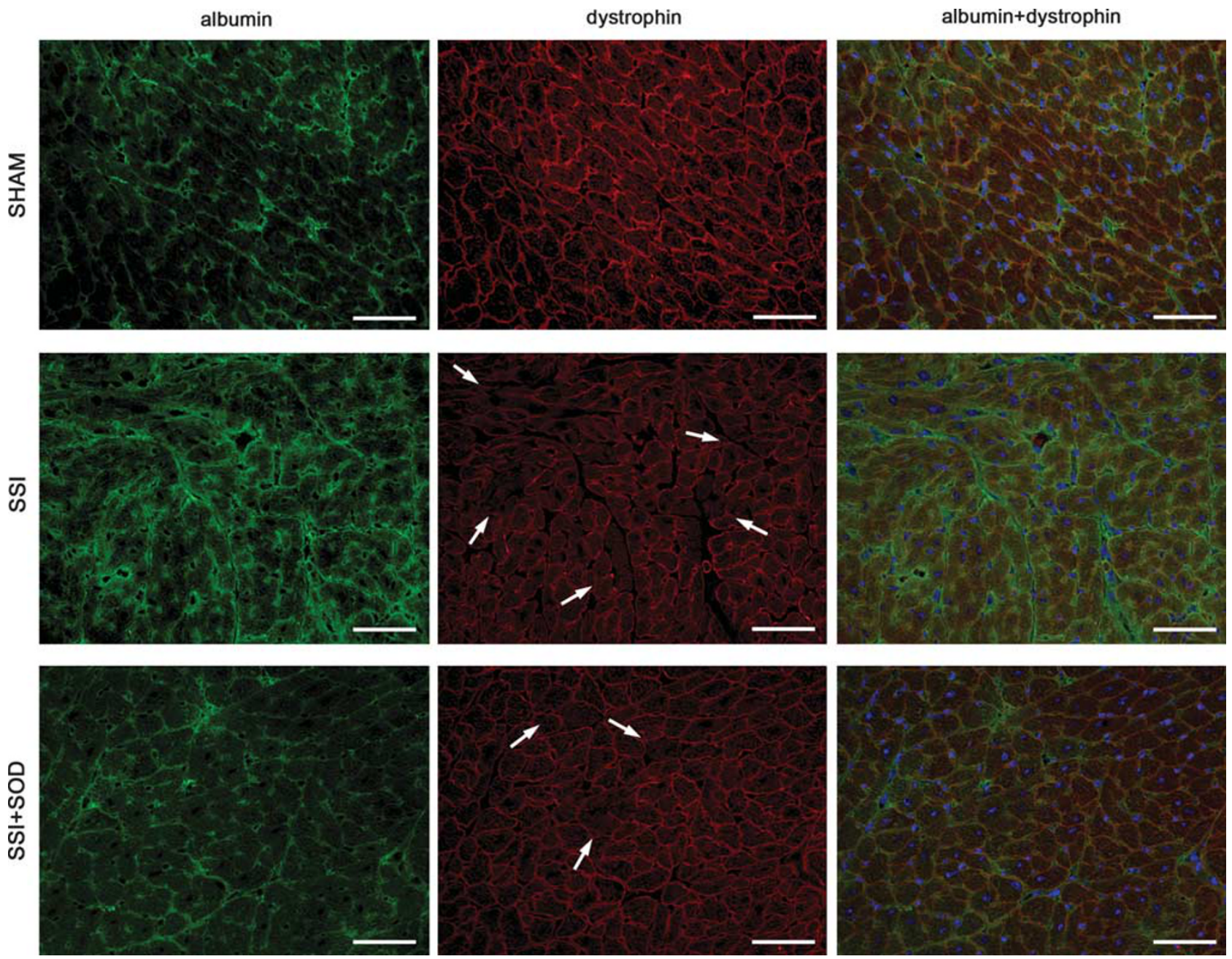

Figure 7 Structural integrity analysis of the sarcolemma. Immunolabeling for albumin (green fluorescence) and dystrophin (red fluorescence). Two different combinations were found: in the sham-operated myocardium and in most of the septic myocardium, total absence of albumin staining in the cytoplasm of myocytes and normal dystrophin labeling indicating absence of plasma membrane damage; in the SSI myocardium, blocks of myocytes showing cytoplasmic albumin staining and absence of dystrophin labeling indicating damage of the plasma membrane. The merge of albumin and dystrophin fluorescent images clearly showed that SSI mice presented accumulation of albumin in spread blocks of cardiomyocytes depleted of sarcolemmal dystrophin. Scale bars indicate $50 \mu \mathrm{m}$.

In conclusion, this study offers novel and mechanistic data to clarify subcellular events in the pathogenesis of cardiac dysfunction in severe sepsis. The main finding was that severe sepsis leads to a marked reduction in membrane localization of dystrophin and $\beta$-dystroglycan in septic cardiomyocytes, a process that may constitute a structural basis of sepsis-induced cardiac depression. In addition, increased sarcolemmal permeability indicates functional impairment of the dystrophin glycoprotein complex in cardiac myofibers. The finding that in vivo antioxidant treatment significantly attenuated the loss of sarcolemmal dystrophin expression and the increased plasma membrane permeability suggests that oxidative damage could mediate the loss of dystrophin and $\beta$-dystroglycan that occurs in septic mice. These abnormal parameters emerge as therapeutic targets and their modulation may provide beneficial effects on future cardiovascular outcomes and mortality in sepsis.

\section{ACKNOWLEDGEMENTS}

The excellent technical assistance of Monica A Abreu, Maria Elena Riul and Lígia B Santoro is gratefully acknowledged. This work was supported by grants from the Fundação de Amparo à Pesquisa do Estado de São Paulo, FAPESP (06/52882-3, 06/59618-0, 07/52556-1, 07/58843-2, and 07/59448-0) and Conselho Nacional de Desenvolvimento Científico e Tecnológico, CNPq.

\section{DISCLOSURE/CONFLICT OF INTEREST}

The authors declare no conflict of interest.

1. Allikian MJ, Mcnally EM. Processing and assembly of the dystrophin glycoprotein complex. Traffic 2007;8:177-183.

2. Davies KE, Nowak KJ. Molecular mechanisms of muscular dystrophies: old and new players. Nat Rev Mol Cell Biol 2006;7:762-773. 
3. Jennings RB, Reimer KA, Steenbergen C. Myocardial ischemia revisited. The osmolar load, membrane damage, and reperfusion. J Mol Cell Cardiol 1986;18:769-780.

4. Parker MM, Shelhamer JH, Bacharach SL, et al. JE: Profound but reversible myocardial depression in patients with septic shock. Ann Intern Med 1984;100:483-490.

5. Parrillo JE, Parker MM, Natanson C, et al. Septic shock in humans. Advances in understanding of pathogenesis, cardiovascular dysfunction, and therapy. Ann Intern Med 1990;113:227-242.

6. Rossi MA, Celes MR, Prado CM, et al. Myocardial structural changes in long-term human severe sepsis/septic shock may be responsible for cardiac dysfunction. Shock 2007;27:10-18.

7. Celes MR, Torres-Dueñas D, Prado CM, et al. Shock 2010, (in press).

8. Wichterman KA, Baue $A E$, Chaudry IH. Sepsis and septic shock: a review of laboratory models and a proposal. J Surg Res 1980;29:189-201.

9. Hubbard WJ, Choudhry M, Schwacha MG, et al. Cecal ligation and puncture. Shock 2005;24:52-57.

10. $\mathrm{Yu} \mathrm{XW}, \mathrm{Chen} \mathrm{Q}$, Kennedy $\mathrm{RH}$, et al. Inhibition of sarcoplasmic reticular function by chronic interleukin-6 exposure via iNOS in adult ventricular myocytes. J Physiol 2005;566:327-340.

11. Zanotti-Cavazzoni SL, Hollenberg SM. Cardiac dysfunction in severe sepsis and septic shock. Curr Opin Crit Care 2009;15:392-397.

12. Standiford TJ, Kunkel SL, Lukacs NW, et al. Macrophage inflammatory protein-1 alpha mediates lung leukocyte recruitment, lung capillary leak, and early mortality in murine endotoxemia. J Immunol 1995;155:1515-1524.

13. Speyer $\mathrm{CL}$, Gao H, Rancilio NJ, et al. Novel chemokine responsiveness and mobilization of neutrophils during sepsis. Am J Pathol 2004;165:2187-2196.

14. Jung C, Martins AS, Niggli E, et al. Dystrophic cardiomyopathy: amplification of cellular damage by $\mathrm{Ca}^{2+}$ signaling and reactive oxygen species-generating pathways. Cardiovasc Res 2008;77:766-773.

15. Danialou G, Comtois AS, Dudley R, et al. Dystrophin-deficient cardiomyocytes are abnormally vulnerable to mechanical stress induced contractile failure and injury. FASEB J 2001;15:1655-1657.

16. Khairallah $M$, Khairallah $R$, Young $M E$, et al. Metabolic and signaling alterations in dystrophin-deficient hearts precede overt cardiomyopathy. J Mol Cell Cardiol 2007;43:119-129.

17. Wu B, Moulton HM, Iversen PL, et al. Effective rescue of dystrophin improves cardiac function in dystrophin-deficient mice by a modified morpholino oligomer. Proc Natl Acad Sci USA 2008;105: 14814-14819.

18. Williamson RA, Henry MD, Daniels KJ, et al. Dystroglycan is essential for early embryonic development: disruption of Reichert's membrane in Dag1-null mice. Hum Mol Genet 1997;6:831-841.

19. Pittet D, Rangel-Frausto $\mathrm{S}$, Li N, et al. Systemic inflammatory response syndrome, sepsis, severe sepsis and septic shock: incidence, morbidities and outcomes in surgical ICU patients. Intensive Care Med 1995;21:302-309.

20. Sgambato A, Brancaccio A. The dystroglycan complex: from biology to cancer. J Cell Physiol 2005;205:163-169.

21. Matsumura $K$, Arai $K$, Zhong D, et al. Disruption of dystroglycan axis by beta-dystroglycan processing in cardiomyopathic hamster muscle. Neuromuscul Disord 2003;13:796-803.
22. Salvemini D, Cuzzocrea S. Oxidative tress in septic shock and disseminated intravascular coagulation. Free Radic Biol Med 2002;33:1173-1185.

23. Cuzzocrea S, Riley DP, Caputi AP, et al. Antioxidant therapy: a new pharmacological approach in shock, inflammation, and ischemia/ reperfusion injury. Pharmacol Rev 2001;53:135-159.

24. Rodríguez M, Cai W-J, Kostin S, et al. Ischemia depletes dystrophin and inhibits protein synthesis in the canine heart: mechanisms of myocardial ischemic injury. J Mol Cell Cardiol 2005;38: 723-733.

25. den Uil CA, Klijn E, Lagrand WK, et al. The microcirculation in health and critical disease. Prog Cardiovasc Dis 2008;51:161-170.

26. Straub V, Bittner RE, Léger JJ, et al. Direct visualization of the dystrophin network on skeletal muscle fiber membrane. J Cell Biol 1992;119:1183-1191.

27. Rybakova IN, Patel JR, Ervasti JM. The dystrophin complex forms a mechanically strong link between the sarcolemma and costameric actin. J Cell Biol 2000;150:1209-1214.

28. Ervasti JM, Ohlendieck K, Kahl SD, et al. Deficiency of a glycoprotein component of the dystrophin complex in dystrophic muscle. Nature 1990;345:315-319.

29. Reggiani C. Between channels and tears: aim at ROS to save the membrane of dystrophic fibres. J Physiol 2008;586:1779.

30. Messina S, Bitto A, Aguennouz M, et al. Nuclear factor kappa-B blockade reduces skeletal muscle degeneration and enhances muscle function in mdx mice. Exp Neurol 2006;198:234-241.

31. Hinshaw LB. Sepsis/septic shock. Participation of the microcirculation: an abbreviated review. Crit Care Med 1996;24:1072-1078.

32. Rossi MA, Santos CS. Sepsis related microvascular myocardial damage with the giant cell inflammation and calcification. Virchows Arch 2003;443:87-92.

33. Chagnon F, Bentourkia M, Lecomte $R$, et al. Endotoxin-induced heart dysfunction in rats: assessment of myocardial perfusion and permeability and the role of fluid resuscitation. Crit Care Med 2006;34:127-133.

34. Campos EC, Romano MM, Prado CM, et al. Isoproterenol induces primary loss of dystrophin in rat hearts: correlation with myocardial injury. Int J Exp Pathol 2008;89:367-381.

35. Armstrong Sc, Latham CA, Shivell CL, et al. Ischemic loss of sarcolemmal dystrophin and spectrin: correlation with myocardial injury. J Mol Cell Cardiol 2001;33:1165-1179.

36. Armstrong SC, Latham CA, Ganote CE. An ischemic betadystroglycan (betaDG) degradation product: correlation with irreversible injury in adult rabbit cardiomyocytes. Mol Cell Biochem 2003;242:71-79.

37. Badorff C, Lee GH, Lamphear BJ, et al. Enteroviral protease $2 \mathrm{~A}$ cleaves dystrophin: evidence of cytoskeletal disruption in an acquired cardiomyopathy. Nat Med 1999;5:320-326.

38. Xiong D, Yajima T, Lim BK, et al. Inducible cardiac-restricted expression of enteroviral protease $2 \mathrm{~A}$ is sufficient to induce dilated cardiomyopathy. Circulation 2007;115:94-102.

39. Whitehead NP, Yeung EW, Allen DG. Muscle damage in mdx (dystrophic) mice: role of calcium and reactive oxygen species. Clin Exp Pharmacol Physiol 2006;33:657-662. 